\title{
Intensivmedizin und Organspende
}

\author{
Gerhard Jorch, Hugo Van Aken
}

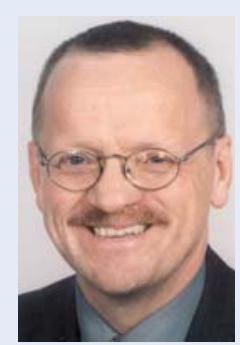

Gerhard Jorch

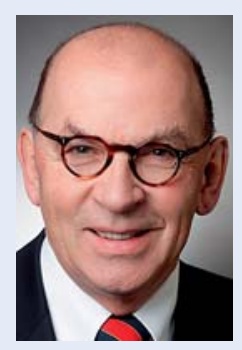

Hugo Van Aken

Korrespondenzadresse

Prof. Dr. Dr. h.c.

Hugo Van Aken

Klinik für Anästhesiologie, operative Intensivmedizin und Schmerztherapie Universitätsklinikum Münster

Albert-Schweitzer-

Campus 1, Gebäude A1

48149 Münster

hva@uni-muenster.de

\section{$\mathrm{H}$}

irntod (irreversibler Hirnfunktionsausfall), Organspende und Transplantation sind in der Intensivmedizin nicht Begriffe theoretischer medizinethischer Diskussionen, sondern praktische Herausforderungen der täglichen Arbeit. Wir diagnostizieren den Hirntod bei Patienten, deren Leben wir nicht retten konnten, und behandeln Patienten, deren Rettung nur durch eine Organtransplantation eines anderen Patienten möglich ist. Organspender und Organempfänger sind gleichermaßen unsere Patienten. Wir sehen von beiden Seiten auf dieses Thema. Mit einem Gefühl der Ohnmacht haben viele von uns in den letzten Jahren miterlebt, wie in der öffentlichen Diskussion ein Akt größter Mitmenschlichkeit - ein Mensch entscheidet über seinen Tod hinaus, durch eine Entnahme seiner Organe das Weiterleben eines anderen Menschen zu ermöglichen Zweifeln und Vorwürfen ausgesetzt wurde.

Wir sollten uns aber nicht allein darauf zurückziehen, dass es sich bei den in den Medien thematisierten „Organspendeskandalen“ um Einzelfälle gehandelt hat. Bei einem derart wichtigen Thema besteht kein Zweifel, dass selbst bei Einzelfällen auf unserer Seite Handlungsbedarf besteht, die Abläufe zu vereinheitlichen, die Kompetenz zu steigern, die Kommunikation zu verbessern und die Transparenz zu erhöhen.

Im Präsidium der Deutschen Interdisziplinären Vereinigung für Intensiv- und Notfallmedizin (DIVI) haben wir uns deshalb Ende 2014 entschlossen, der im Auftrag des Bundesministeriums für Gesundheit (BMG) tätigen Bundesärztekammer (BÄK) unsere Mitwirkung bei der Umsetzung des Transplantationsgesetzes von 1997, novelliert 2012, anzubieten. Dieses geschah in enger Abstimmung und im Konsens mit den Vorständen der bereits zuvor involvierten Deutschen Transplantationsgesellschaft (DTG) und der Deutschen Stiftung Organtransplantation (DSO). Mitglieder der wissenschaftlichen Sektionen der DIVI Organspende und -transplantation, Ethik, Koma und Neuromedizinstandards wurden von der Ständigen Kommission Organtransplantation (StäKo) der BÄK für die Amtsperiode 2015-19 in die Arbeitsgruppen „Spenderbeurteilung und Empfängerschutz“ (Federführend: Prof. Klaus Hahnenkamp/Greifswald) und „Transplantationsbeauf- tragte“ (Federführend: Frau Nadja Komm/Heidelberg) berufen und haben bereits ihre Arbeit aufgenommen.

Als Kernpunkte der Arbeit stellen sich bereits jetzt die Analyse und Diskussion der Behandlung von Intensivpatienten mit infauster Prognose und Kommunikation mit den Angehörigen vor Eintritt des Hirntodes sowie eine bessere Einbindung des für die sogenannten Entnahmekliniken im Gesetz vorgeschriebenen Transplantationsbeauftragten in das intensivmedizinische Management dar. Beide Ziele können nur durch hohes Engagement und Kooperation des Intensivbehandlungsteams erreicht werden.

Am 30. März 2015 genehmigte das Bundesministerium für Gesundheit die 4. Fortschreibung der Richtlinie zur Feststellung des irreversiblen Hirnfunktionsausfalls gemäß §16 Abs. 3 Transplantationsgesetz (TPG) vorgelegt vom Arbeitskreis „Fortschreibung der Richtlinien zur Feststellung des Hirntodes“ des Wissenschaftlichen Beirats der BÄK. Sie beruht nach wie vor auf den bereits 1982 erstmalig vom Wissenschaftlichen Beirat der BÄK formulierten Prinzipien. Allerdings wurden einige für die Ausführung relevante Details präzisiert. Die wichtigsten sind, dass die Hirntodfeststellung durch zwei voneinander unabhängige in der Intensivmedizin erfahrene Fachärzte erfolgt, mindestens einer hiervon muss Facharzt für Neurologie oder Neurochirurgie sein. Bei Patienten vor Vollendung des 14. Lebensjahres muss einer der zwei Ärzte Facharzt für Kinder- und Jugendmedizin sein. Wenn dieser Facharzt für Kinderund Jugendmedizin Neuropädiater ist, erübrigt sich die Hinzuziehung eines Neurologen oder Neurochirurgen.

Wenngleich bisher kein Fall eines fälschlicherweise diagnostizierten Hirntodes bekannt ist, die Diagnostik also bislang in der Praxis sicher war, ist aus Gründen der Akzeptanz und Glaubwürdigkeit in Zukunft verstärkt darauf zu achten, dass alle formalen Festlegungen eingehalten werden. Dabei trägt der/die Transplantationsbeauftragte eine zentrale Verantwortung. Um ihr gerecht zu werden, benötigt er/sie die volle Unterstützung der auf den Intensivstationen tätigen Schwestern, Ärzte und Pfleger und eine professionelle Einbindung in die SOPs der einzelnen Häuser. 